Supporting Information

\title{
Utica Shale Play Oil and Gas Brines: Geochemistry and Factors Influencing Wastewater Management
}

\author{
Madalyn S. Blondes ${ }^{1 *}$, Jenna L. Shelton ${ }^{1}$, Mark A. Engle ${ }^{2}$, Jason P. Trembly ${ }^{3}$, Colin A. Doolan ${ }^{1}$, \\ Aaron M. Jubb ${ }^{1}$, Jessica C. Chenault ${ }^{1}$, Elisabeth L. Rowan ${ }^{1 \#}$, Ralph J. Haefner ${ }^{4}$, Brian E Mailot ${ }^{5}$ \\ ${ }^{1}$ U.S. Geological Survey, Eastern Energy Resources Science Center, 12201 Sunrise Valley Dr., MS956, Reston, VA \\ 20192, United States \\ ${ }^{2}$ The University of Texas at El Paso, Department of Geological Sciences, 500 W. University Ave., El Paso, TX \\ 79968, United States \\ ${ }^{3}$ Ohio University, Institute for Sustainable Energy and the Environment, 259 Stocker Center, Athens OH 45701, \\ United States \\ ${ }^{4}$ U.S. Geological Survey, Upper Midwest Water Science Center, 5840 Enterprise Drive Lansing, MI 48911, United \\ States \\ ${ }^{5}$ U.S. Geological Survey, Ohio-Kentucky-Indiana Water Science Center, 6460 Busch Blvd. Ste. 100, Columbus, \\ Ohio 43229, United States \\ *Corresponding author: mblondes@usgs.gov, (703) 648-6509 \\ ${ }^{\#}$ Retired
}

Supporting Information for:

Blondes, M. S.; Shelton, J. L.; Engle, M. A.; Trembly, J. P.; Doolan, C. A.; Jubb, A. M.; Chenault, J. M.; Rowan, E. L.; Haefner, R. J.; Mailot, B. E. Utica Shale Play Oil and Gas Brines: Geochemistry and Factors Influencing Wastewater Management. Environ. Sci.

Technol. 2020. https://doi.org/10.1021/acs.est.0c02461.

Pages: 10

Figures: 2

Tables: 2 


\section{Stratigraphic Column with Sample Locations}

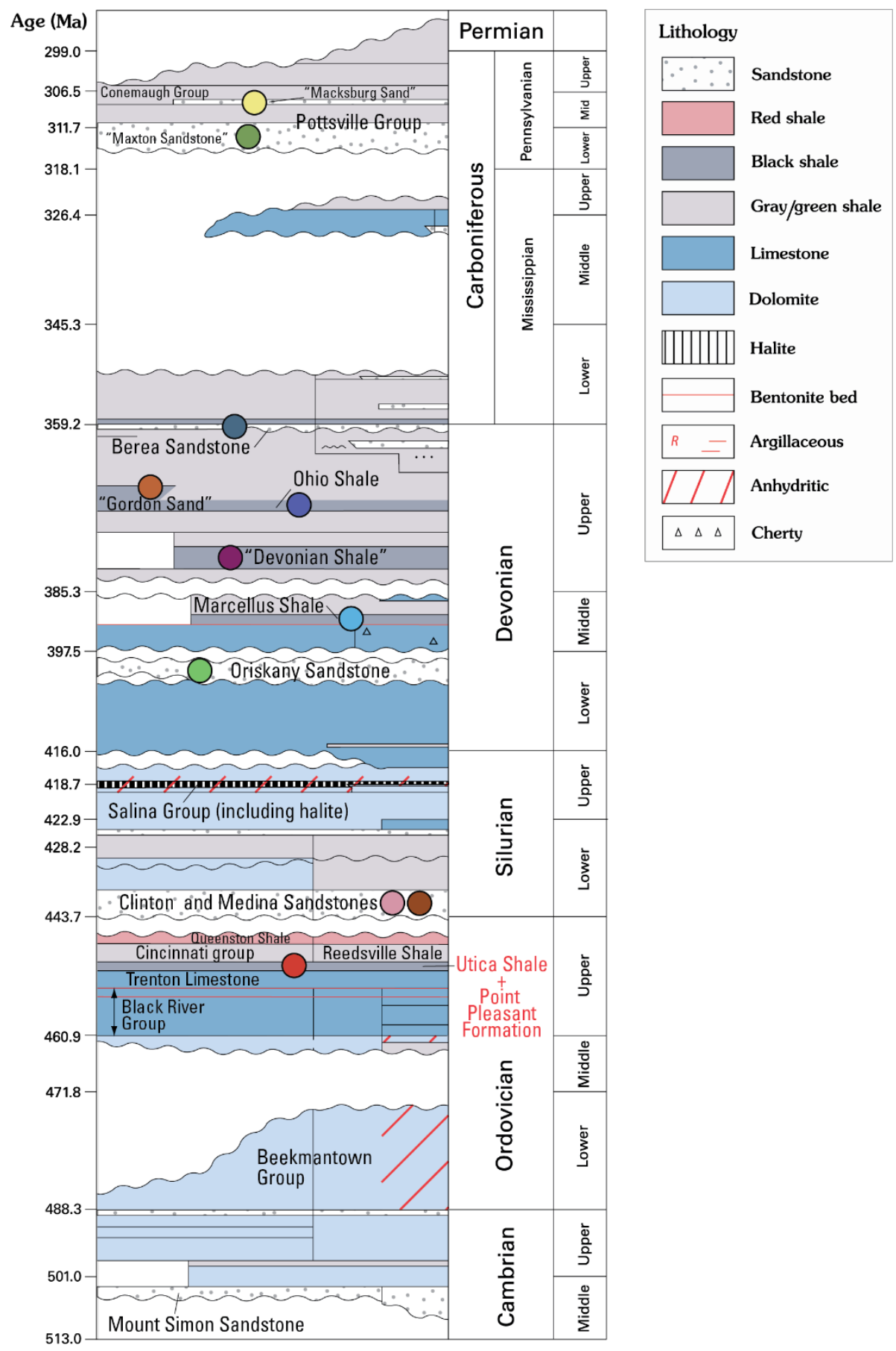

Figure S1. Stratigraphic column in eastern Ohio and western Pennsylvania, modified from Ryder et al. ${ }^{1}$ Color-filled circles represent the formation from which samples were collected, as shown in Figures 1,3, and 5. Formations in quotes are drillers' terms. 


\section{BRInE Lab QA/QC}

\section{Major and Trace Cations}

Analytical precision for elements (major and trace cations) measured using the Horiba Ultima Expert inductively coupled plasma - optical emission spectrometer (ICP-OES) was determined by propagating the standard deviation from triplicate measurements of an analytical emission line through a linear regression fit (weighted to the standard deviation at each point) to a log-log plot of emission intensity versus concentration for a series of five calibration standards with known element concentrations. The analytical precision determined in this fashion was typically better than $\pm 5 \%$ at the $2 \sigma$ level. Quality control samples included in each analytical run on the ICP-OES included blanks, calibration standards, surrogate reference materials [e.g., certified seawater from High Purity Standards (North Charleston, South Carolina)], laboratory duplicates, laboratory replicates, and matrix spikes. Total measurement uncertainty for major and trace cations is estimated at $\pm 30 \%$ at the $2 \sigma$ level based on matrix spike recoveries. For more information see Jubb et al. ${ }^{2}$

\section{Anions}

Analytical precision for anions $\left(\mathrm{Cl}^{-}, \mathrm{Br}^{-}\right.$, and $\left.\mathrm{SO}_{4}{ }^{2-}\right)$ measured using U.S. Environmental Protection Agency method $9056 \mathrm{~A}^{3}$ was determined by propagating the standard deviation from duplicate measurements of chromatographic peak area through a linear regression fit (weighted to the standard deviation at each point) to a log-log plot of peak area versus concentration for a series of five calibration standards with known anion concentrations. The analytical precision determined in this fashion was typically better than $\pm 2 \%$ at the $2 \sigma$ level. Quality control samples included in each analytical run on the ion chromatograph included blanks, calibration standards, surrogate reference materials [e.g., U.S. Geological Survey standard reference samples (https://bqs.usgs.gov/srs/)], laboratory duplicates, laboratory replicates, and matrix spikes. Total measurement uncertainty for anions is estimated at $\pm 20 \%$ at the $2 \sigma$ level based on matrix spike recoveries.

\section{Total Dissolved Solids (TDS) and Specific Gravity}

Quality control samples for TDS and specific gravity determinations using EPA method 160.1 ${ }^{4}$ and ASTM method D1429-13, ${ }^{5}$ respectively, included blanks, laboratory duplicates, laboratory replicates, and laboratory control standards (LCSs) consisting of 5\% wt. and $10 \% \mathrm{wt} . \mathrm{NaCl}$ solutions. Total uncertainty of TDS determinations is estimated at $\pm 30 \%$ at the $2 \sigma$ level based on LCS recoveries. Total uncertainty of specific gravity determinations is estimated at $\pm 2 \%$ at the $2 \sigma$ level based on LCS recoveries. 


\section{Tables of Results}

The following data tables present data discussed and plotted in the main manuscript. The data are also available as a U.S. Geological Survey Data Release. ${ }^{6}$ 
Table S1. Produced water sample location and descriptive information, physical parameters, and isotopes.

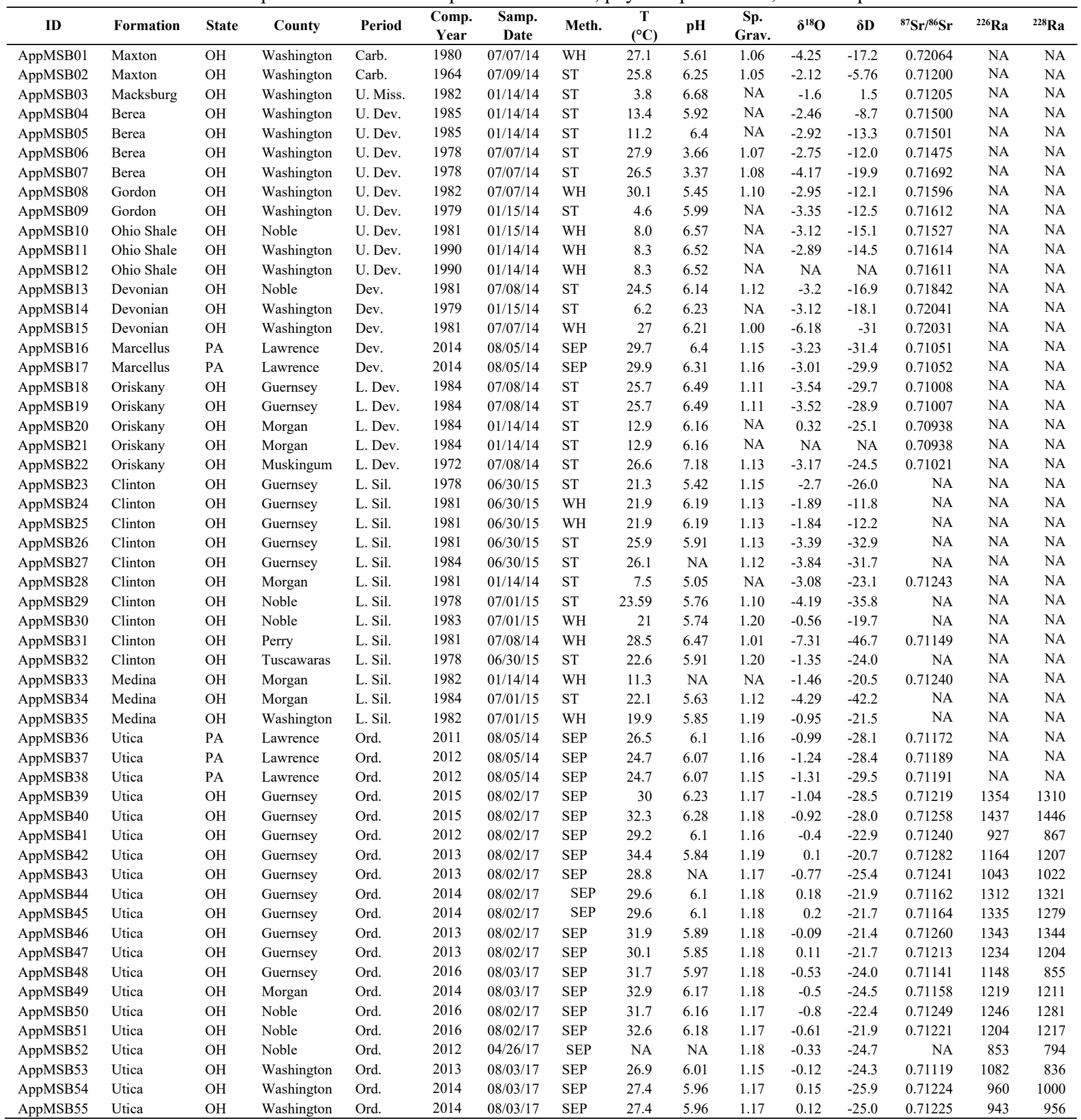

Comp. $=$ completion, Samp. $=$ sample, Cond. $=$ conductivity, Meth. $=$ sample method, $\mathrm{T}=$ temperature at surface,

Sp. Grav. $=$ specific gravity. ND is an instrument non-detect. $\mathrm{Carb}=$ Carboniferous, Miss $=$ Mississippian, Dev $=$ Devonian, $\mathrm{Sil}=$ Silurian, $\mathrm{Ord}=$ Ordovician, $\mathrm{WH}=$ wellhead, $\mathrm{ST}=$ stock tank, $\mathrm{SEP}=$ separator, NA represents a not analyzed sample. Proprietary individual Utica Shale Play sample depths range from 6,700 ft to 8,600 ft. 
Table S2. Produced water major and trace element composition.

\begin{tabular}{|c|c|c|c|c|c|c|c|c|c|c|c|c|c|c|c|c|}
\hline ID & Formation & TDS & $\mathrm{Ca}$ & $\mathbf{M g}$ & $\mathbf{K}$ & $\mathrm{Na}$ & $\mathrm{Br}$ & $\mathrm{Cl}$ & $\mathrm{SO}_{4}$ & $\mathbf{B a}$ & B & $\mathrm{Fe}$ & $\mathbf{L i}$ & Mn & $\mathbf{S i}$ & $\mathrm{Sr}$ \\
\hline AppMSB01 & Maxton & 86000 & 7060 & 1000 & 126 & 25900 & 588 & 51800 & 37.9 & 57.1 & 0.266 & 90.6 & 3.55 & 2.97 & 2.54 & 104 \\
\hline AppMSB02 & Maxton & 63000 & 3310 & 848 & 38.5 & 20100 & 369 & 39100 & 42.8 & 19.5 & 0.433 & 6.67 & 0.370 & 2.65 & 0.390 & 237 \\
\hline AppMSB03 & Macksburg & 52600 & 3310 & 735 & 51.9 & 13700 & 186 & 27600 & 1230 & 0.079 & 0.211 & 41.9 & 0.458 & 1.62 & 4.47 & 134 \\
\hline AppMSB04 & Berea & 144000 & 11600 & 2520 & 161 & 39300 & 662 & 91600 & $\mathrm{ND}$ & 44 & 0.556 & 51.4 & 2.46 & 8.66 & 6.50 & 241 \\
\hline AppMSB05 & Berea & 166000 & 13300 & 2840 & 177 & 46100 & 780 & 115000 & ND & 44.5 & 0.53 & 79.6 & 2.73 & 10.8 & 9.12 & 297 \\
\hline AppMSB06 & Berea & 96000 & 5680 & 1270 & 112 & 27200 & 600 & 57800 & 46.5 & 141 & 0.540 & 1.60 & 1.84 & 4.61 & 0.964 & 110 \\
\hline AppMSB07 & Berea & 112000 & 10400 & 1880 & 128 & 32100 & 780 & 69600 & 48.1 & 68.0 & 0.340 & 244 & 2.64 & 23.2 & ND & 157 \\
\hline AppMSB08 & Gordon & 136000 & 9960 & 2100 & 143 & 38700 & 841 & 81400 & 58.6 & 82.2 & 0.585 & 97.0 & 4.47 & 8.38 & ND & 168 \\
\hline AppMSB09 & Gordon & 126000 & 10700 & 2070 & 152 & 33900 & 692 & 92300 & ND & 126 & ND & 78.5 & 2.52 & 9.91 & 5.47 & 179 \\
\hline AppMSB10 & Ohio Shale & 95400 & 6110 & 1370 & 118 & 27800 & 424 & 58700 & ND & 208 & 0.729 & 70.7 & 2.63 & 5.32 & 9.72 & 118 \\
\hline AppMSB11 & Ohio Shale & 128000 & 9930 & 2050 & 143 & 34100 & 611 & 77300 & ND & 84.8 & 0.738 & 67.7 & 4.04 & 9.47 & 9.35 & 166 \\
\hline AppMSB12 & Ohio Shale & 128000 & 10200 & 2060 & 128 & 34700 & 620 & 77300 & ND & 83.1 & 0.71 & 67 & 4.15 & 9.34 & 3.32 & 164 \\
\hline AppMSB13 & Devonian & 167000 & 18600 & 3290 & 185 & 49700 & 1270 & 102000 & 73.7 & 68.9 & 1.47 & 205 & 8.45 & 19.7 & 1.30 & 238 \\
\hline AppMSB14 & Devonian & 134000 & 14100 & 2380 & 162 & 33800 & 947 & 87200 & ND & 127 & 2.3 & 334 & 5.35 & 25.2 & 9.30 & 177 \\
\hline AppMSB15 & Devonian & 4000 & 63.1 & 26.4 & 6.81 & 1520 & 25.6 & 2560 & ND & 0.304 & 0.254 & 2.88 & 0.202 & 0.133 & 2.40 & 0.811 \\
\hline AppMSB16 & Marcellus & 212000 & 11700 & 1300 & 472 & 35300 & 1810 & 128000 & 96.1 & 34.4 & 5.69 & 39.0 & 104 & 2.60 & 4.44 & 2400 \\
\hline AppMSB17 & Marcellus & 223000 & 22200 & 2230 & 817 & 56600 & 1900 & 134000 & 102 & 143 & 9.29 & 109 & 176 & 6.57 & 6.93 & 4160 \\
\hline AppMSB18 & Oriskany & 161000 & 10600 & 2320 & 1060 & 47100 & 1180 & 95900 & 148 & 1.35 & 27.0 & 160 & 88.7 & 1.55 & 2.34 & 857 \\
\hline AppMSB19 & Oriskany & 158000 & 10400 & 2160 & 1000 & 44100 & 1130 & 98000 & 148 & 1.20 & 25.2 & 154 & 84.0 & 1.44 & 1.89 & 820 \\
\hline AppMSB20 & Oriskany & 259000 & 23300 & 4480 & 2990 & 76100 & 1600 & 183000 & 182 & 1.78 & 133 & 92.7 & 61 & 5.29 & 13.8 & 775 \\
\hline AppMSB21 & Oriskany & 259000 & 22900 & 4440 & 2940 & 70400 & 1560 & 179000 & 155 & 1.45 & 130 & 82.1 & 63 & 5.65 & 28.4 & 764 \\
\hline AppMSB22 & Oriskany & 191000 & 14200 & 2820 & 942 & 50200 & 1440 & 116000 & 82.8 & 7.23 & 17.9 & 138 & 81.9 & 1.63 & 0.646 & 1040 \\
\hline AppMSB23 & Clinton & 212000 & 19500 & 1680 & 1290 & 52800 & 1430 & 109000 & 99.4 & 2.12 & 11.5 & 220 & 69.4 & 35.7 & 2.12 & 611 \\
\hline AppMSB24 & Clinton & 188000 & 13400 & 2790 & 534 & 46200 & 1070 & 94200 & 49.3 & 40.2 & 3.94 & 233 & 15.9 & 16.9 & ND & 403 \\
\hline AppMSB25 & Clinton & 185000 & 15000 & 3040 & 561 & 48400 & 1060 & 93600 & 44.5 & 45.6 & 3.88 & 254 & 17.0 & 19.1 & ND & 440 \\
\hline AppMSB26 & Clinton & 194000 & 24900 & 2670 & 1360 & 43700 & 1510 & 111000 & 103 & 6.95 & 13.3 & 261 & 49.4 & 29.0 & 0.570 & 725 \\
\hline AppMSB27 & Clinton & 170000 & 24900 & 2250 & 1170 & 41800 & 1390 & 98200 & 157 & 1.00 & 7.62 & 253 & 43.1 & 38.2 & ND & 772 \\
\hline AppMSB28 & Clinton & 251000 & 28900 & 3680 & 1820 & 58300 & 3300 & 190000 & 169 & 1.59 & 21.3 & 132 & 49.2 & 38.4 & 9.72 & 1150 \\
\hline AppMSB29 & Clinton & 147000 & 13100 & 1180 & 940 & 34000 & 1090 & 77400 & 28.0 & 0.390 & 8.28 & 256 & 42.6 & 23.8 & 0.079 & 490 \\
\hline AppMSB30 & Clinton & 278000 & 27400 & 2010 & 2150 & 72000 & 2150 & 142000 & 282 & 2.20 & 21.1 & 164 & 103 & 57.3 & 2.66 & 1010 \\
\hline AppMSB31 & Clinton & 8000 & 327 & 76.0 & 21.9 & 2030 & 49.5 & 5420 & 66.8 & 0.538 & 0.263 & 12.2 & 0.356 & 0.443 & 1.23 & 13.5 \\
\hline AppMSB32 & Clinton & 290000 & 33100 & 2770 & 2200 & 80300 & 2440 & 169000 & 359 & 1.86 & 20.0 & 178 & 108 & 53.6 & 2.71 & 1070 \\
\hline AppMSB33 & Medina & 265000 & 32300 & 4040 & 1990 & 62000 & 3010 & 186000 & 135 & 2.85 & 14.4 & 190 & 58 & 50.7 & 12.6 & 1070 \\
\hline AppMSB34 & Medina & 176000 & 13300 & 1270 & 1150 & 36000 & 1160 & 85400 & 27.0 & 3.10 & 10.8 & 237 & 40.8 & 27.6 & ND & 469 \\
\hline AppMSB35 & Medina & 288000 & 28500 & 3210 & 1650 & 65200 & 1920 & 143000 & 231 & 1.89 & 13.8 & 77.1 & 74.2 & 42.9 & ND & 889 \\
\hline AppMSB36 & Utica & 229000 & 21700 & 2340 & 939 & 57900 & 2060 & 131000 & 84.4 & 2810 & 6.74 & 116 & 72.0 & 16.1 & 12.1 & 4170 \\
\hline AppMSB37 & Utica & 219000 & 11900 & 1360 & 649 & 32600 & 2010 & 129000 & 71.3 & 1290 & 6.84 & 81.7 & 37.2 & 7.05 & 7.09 & 2510 \\
\hline AppMSB38 & Utica & 214000 & 21600 & 2410 & 1162 & 56200 & 1960 & 128000 & 86.4 & 2250 & 12.2 & 146 & 65.3 & 12.8 & 12.2 & 4190 \\
\hline AppMSB39 & Utica & 258000 & 31900 & 3190 & 697 & 58600 & 1850 & 145000 & 128 & 46.4 & 6.59 & 131 & 47.7 & 33.8 & 10.9 & 3010 \\
\hline AppMSB40 & Utica & 268000 & 35800 & 3330 & 742 & 63200 & 2090 & 155000 & 139 & 51.8 & 6.52 & 139 & 50.4 & 44.7 & 11.6 & 3160 \\
\hline AppMSB41 & Utica & 243000 & 37000 & 3280 & 694 & 56600 & 1790 & 132000 & 144 & 22.7 & 6.90 & 273 & 45.9 & 27.9 & 15.4 & 2740 \\
\hline AppMSB42 & Utica & 283000 & 41600 & 3450 & 820 & 60900 & 2050 & 152000 & 138 & 33.0 & 6.74 & 219 & 51.3 & 51.3 & 11.7 & 3140 \\
\hline AppMSB43 & Utica & 249000 & 33200 & 3200 & 727 & 58400 & 2080 & 150000 & 94.9 & 29.7 & 6.90 & 162 & 48.2 & 31.0 & 11.3 & 3030 \\
\hline AppMSB44 & Utica & 268000 & 31200 & 3110 & 611 & 50500 & 2190 & 158000 & 107 & 43.3 & 7.76 & 116 & 39.7 & 27.0 & 9.61 & 3070 \\
\hline AppMSB45 & Utica & 258000 & 37400 & 3410 & 665 & 53400 & 2170 & 155000 & 103 & 48.0 & 8.80 & 132 & 42.1 & 30.5 & 12.1 & 3460 \\
\hline AppMSB46 & Utica & 268000 & 31200 & 2590 & 606 & 44400 & 2000 & 151000 & 134 & 30.4 & 4.75 & 118 & 36.6 & 30.3 & 9.65 & 2300 \\
\hline AppMSB47 & Utica & 264000 & 37100 & 3710 & 838 & 63700 & 2070 & 153000 & 130 & 43.2 & 6.96 & 164 & 54.5 & 32.1 & 10.8 & 3520 \\
\hline AppMSB48 & Utica & 268000 & 34100 & 3890 & 1160 & 52600 & 2290 & 168000 & 219 & 8.96 & 16.8 & 90.6 & 40.5 & 9.60 & 6.94 & 2010 \\
\hline AppMSB49 & Utica & 262000 & 36600 & 3900 & 802 & 54500 & 2020 & 159000 & 223 & 12.8 & 6.35 & 155 & 44.5 & 15.1 & 11.4 & 2500 \\
\hline AppMSB50 & Utica & 245000 & 30100 & 2820 & 770 & 58100 & 2020 & 150000 & 141 & 31.6 & 7.22 & 104 & 47.3 & 37.6 & 11.3 & 2580 \\
\hline AppMSB51 & Utica & 240000 & 27700 & 2420 & 640 & 45100 & 1890 & 147000 & 151 & 33.8 & 8.07 & 86.5 & 36.3 & 27.3 & 11.1 & 2240 \\
\hline AppMSB52 & Utica & 251000 & 41100 & 4820 & 736 & 55800 & 2000 & 153000 & 284 & 10.4 & 6.04 & 147 & 40.7 & 15.3 & 5.76 & 3070 \\
\hline AppMSB53 & Utica & 237000 & 34700 & 3760 & 1010 & 54100 & 1920 & 142000 & 75.1 & 46.5 & 15.8 & 92.6 & 47.4 & 10.4 & 13.6 & 3550 \\
\hline AppMSB54 & Utica & 253000 & 30400 & 3260 & 969 & 61000 & 2160 & 160000 & 52.0 & 102 & 8.14 & 147 & 59.8 & 16.2 & 13.1 & 3260 \\
\hline AppMSB55 & Utica & 263000 & 29400 & 3060 & 899 & 58800 & 2160 & 157000 & 52.9 & 92.3 & 7.95 & 141 & 53.6 & 15.4 & 12.1 & 3010 \\
\hline
\end{tabular}

All units are $\mathrm{mg} / \mathrm{L} . \mathrm{ND}=$ Non-Detect. 


\section{Cluster Analysis}

To determine whether produced water samples cluster into groups, particularly those that seem visually apparent in the ${ }^{87} \mathrm{Sr} /{ }^{86} \mathrm{Sr}$ vs $\mathrm{Sr}$ plot (Figure 3b), we performed a k-means cluster analysis on a subset of samples where $\mathrm{Na}, \mathrm{Ca}, \mathrm{Mg}, \mathrm{Cl}, \mathrm{Sr}$, and ${ }^{87} \mathrm{Sr} /{ }^{86} \mathrm{Sr}$ data all exist. Since the data are compositional, we first pre-treated the data with the centered log ratio (clr) (Aitchison, 1986). We then applied the k-means algorithm using the stats package in $\mathrm{R}$ assuming three clusters. ${ }^{7}$ Results from Figure S1 show that the three general clusters are found in the 1) deep basin brines from reservoirs including the Marcellus, Utica, and those down section, 2) shallower oil field brines, and 3) shallow drinking water wells. Figure $3 \mathrm{~b}$ is reproduced here from the text for comparison.
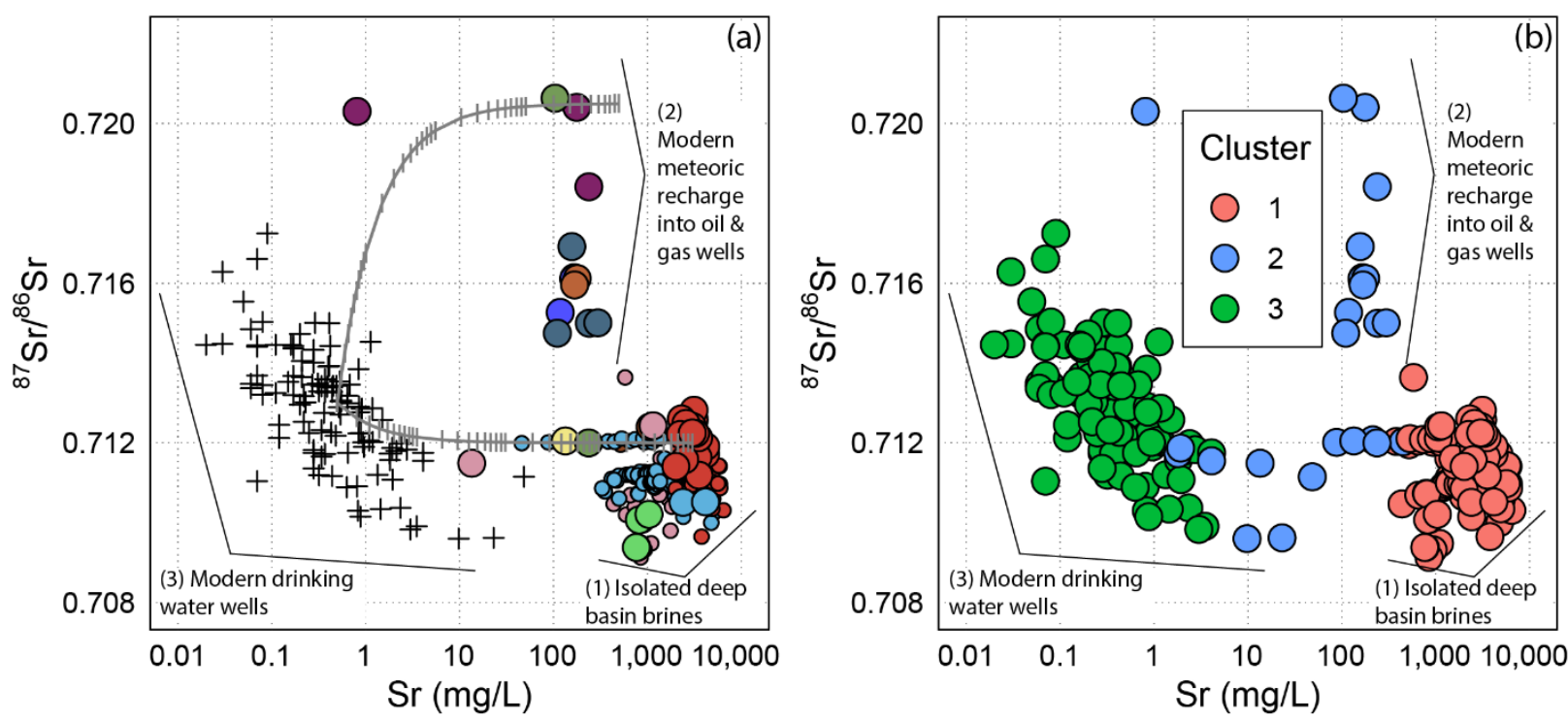

Figure S2. K-means cluster analysis of Appalachian Basin produced water data. (a) Reproduction of Figure $3 b$ for comparison. See Figure 3 for legend. (b) 3 cluster k-means classification. This figure was created using R. ${ }^{7}$ 


\section{Radium secular equilibrium}

We can estimate the ${ }^{228} \mathrm{Ra} /{ }^{226} \mathrm{Ra}$ ratio of water in secular equilibrium with the Utica Shale interbedded K-bentonite layers, and adjacent reservoirs. Because the solubility of Ra is much higher than $\mathrm{Th}$ and $\mathrm{U}$ in reducing, low sulfate waters, ${ }^{8}$ we assume no excess $\mathrm{Ra}$ in the water, i.e., that all $\mathrm{Ra}$ in the water formed from radioactive decay in the shale and then dissolved into the water. We also assume that the solubility of ${ }^{226} \mathrm{Ra}$ and ${ }^{228} \mathrm{Ra}$ are the same such that the ${ }^{228} \mathrm{Ra} /{ }^{226} \mathrm{Ra}$ ratio would be the same in the water as in the rock. At secular equilibrium, the activity of the parent isotope equals the activity of the daughter isotope:

$$
\begin{aligned}
& \lambda_{238_{\mathrm{U}}} N^{238} \mathrm{U}=\lambda^{22{ }_{\mathrm{Ra}}}{ }^{226}{ }_{\mathrm{Ra}} \quad \text { (Eq. S1) } \\
& \lambda_{232}{ }_{\mathrm{Th}}{ }^{N 232} \mathrm{Th}=\lambda_{228}{ }_{\mathrm{Ra}}{ }^{228} \mathrm{Ra} \quad \text { (Eq. S2) }
\end{aligned}
$$

Where $\lambda$ are the decay constants $\left(\lambda_{238_{U}}=1.511 \times 10^{-10}, \lambda_{238_{U}}=4.948 \times 10^{-11}\right){ }^{9-11}, \mathrm{~N}$ is the number of atoms, and $\lambda \mathrm{N}$ is the activity. Therefore, the expected ${ }^{228} \mathrm{Ra} /{ }^{226} \mathrm{Ra}$ activity ratio is:

$$
\frac{{ }^{228} \mathrm{Ra}}{{ }^{226} \mathrm{Ra}}=\frac{\lambda^{232} \mathrm{Th}}{\lambda_{238} \mathrm{U}} \times \frac{{ }^{232} \mathrm{Th}}{N^{238} \mathrm{U}}
$$

If we further assume that the ${ }^{238} \mathrm{U} /{ }^{232} \mathrm{Th}$ atomic ratio can be approximated by the U/Th concentration ratio in the rock, and that this is the U/Th ratio released at the mineral surface, then the expected ${ }^{228} \mathrm{Ra} /{ }^{226} \mathrm{Ra}$ activity ratio is:

$$
\frac{{ }^{228} \mathrm{Ra}}{{ }^{226} \mathrm{Ra}}=\frac{\lambda^{232} \mathrm{Th}}{\lambda^{238} \mathrm{U}} \times \frac{[\mathrm{Th}]}{[\mathrm{U}]}
$$




\section{References}

(1) Ryder, R. T.; Crangle Jr., R. D.; Trippi, M. H.; Swezey, C. S.; Lentz, E. E.; Rowan, E. L.; Hope, R. S. Geologic Cross Section D-D' Through the Appalachian Basin from the Findlay Arch, Sandusky County, Ohio, to the Valley and Ridge Province, Hardy County, West Virginia; 2009. https://doi.org/10.3133/sim3067.

(2) Jubb, A. M.; Engle, M. A.; Chenault, J. M.; Blondes, M. S.; Danforth, C. G.; Doolan, C.; Gallegos, T. J.; Mueller, D.; Shelton, J. L. Direct Trace Element Determination in Oil and Gas Produced Waters with Inductively Coupled Plasma-Optical Emission Spectrometry: Advantages of High Salinity Tolerance. Geostand. Geoanalytical Res. 2020. https://doi.org/10.1111/ggr.12316.

(3) U.S. Environmental Protection Agency (EPA). SW-846 Test Method 9056A:

Determination of Inorganic Anions by Ion Chromatography; 2007.

(4) U.S. Environmental Protection Agency (EPA). Method 160.1 Total Dissolved Solids (TDS) Gravimetric, Dried at 180 Degrees C; 1999.

(5) ASTM. D1429-13, Standard Test Methods for Specific Gravity of Water and Brine. ASTM International, West Conshohocken, PA. 2013.

(6) Blondes, M. S.; Shelton, J. L.; Engle, M. A.; Trembly, J. P.; Doolan, C. A.; Jubb, A. M.; Chenault, J. M.; Rowan, E. L.; Haefner, R. J.; Mailot, B. E. Geochemistry of Utica Shale Play and Other Appalachian Produced Waters. U.S. Geological Survey Data Release 2020. https://doi.org/10.5066/P9AN5GJ0.

(7) R Core Team. R: A Language and Environmental for Statistical Computing. R Foundation 
for Statistical Computing: Vienna, Austria 2019.

(8) Kraemer, T. F.; Reid, D. F. The Occurrence and Behavior of Radium in Saline Formation Water of the U.S. Gulf Coast Region. Chem. Geol. 1984, 46 (2), 153-174. https://doi.org/10.1016/0009-2541(84)90186-4.

(9) Le Roux, L. J. and; Glendenin, L. E. Half-Life of ${ }^{232}$ Th. In Proceedings of the National Meeting on Nuclear Energy, Pretoria, South Africa; 1963; Vol. 83, p 94.

(10) Jaffey, A. H.; Flynn, K. F.; Glendenin, L. E.; Bentley, W. C.; Essling, A. M. Precision Measurement of Half-Lives and Specific Activities of ${ }^{235} \mathrm{U}$ and ${ }^{238}$ U. Phys. Rev. C 1971, 4 (5), 1889-1906. https://doi.org/10.1103/PhysRevC.4.1889.

(11) Sims, K. W. W.; Pichat, S.; Reagan, M. K.; Kyle, P. R.; Dulaiova, H.; Dunbar, N. W.; Prytulak, J.; Sawyer, G.; Layne, G. D.; Blichert-Toft, J.; Gauthier, P. J.; Charette, M. A.; Elliott, T. R. On the Time Scales of Magma Genesis, Melt Evolution, Crystal Growth Rates and Magma Degassing in the Erebus Volcano Magmatic System Using the ${ }^{238} \mathrm{U}$, ${ }^{235} \mathrm{U}$ and ${ }^{232}$ Th Decay Series. J. Petrol. 2012, 54 (2), 235-271. https://doi.org/10.1093/petrology/egs068. 